\title{
Animer et jouer : rencontres et confusions
}

Play and animation, some commonplaces in questions

\section{Baptiste Besse-Patin et Nathalie Roucous}

\section{(2) OpenEdition}

Journals

Édition électronique

URL : https://journals.openedition.org/sdj/1965

DOI : $10.4000 /$ sdj. 1965

ISSN : 2269-2657

\section{Éditeur}

Laboratoire EXPERICE - Centre de Recherche Interuniversitaire Expérience Ressources Culturelles Education

\section{Référence électronique}

Baptiste Besse-Patin et Nathalie Roucous, «Animer et jouer : rencontres et confusions », Sciences du jeu [En ligne], 12 | 2019, mis en ligne le 15 décembre 2019, consulté le 12 décembre 2022. URL : http:// journals.openedition.org/sdj/1965; DOI : https://doi.org/10.4000/sdj.1965

Ce document a été généré automatiquement le 12 décembre 2022.

\section{c) () $९$}

Creative Commons - Attribution - Pas d'Utilisation Commerciale - Pas de Modification 4.0 International - CC BY-NC-ND 4.0

https://creativecommons.org/licenses/by-nc-nd/4.0/ 


\title{
Animer et jouer : rencontres et confusions
}

\author{
Play and animation, some commonplaces in questions
}

Baptiste Besse-Patin et Nathalie Roucous

1 Ce dossier vient interroger la rencontre entre deux objets : le jeu, bien évidemment, et l'animation. Au cours des deux ans de gestation un cheminement s'est opéré pour explorer leurs croisements, mais aussi pour mesurer les difficultés de la thématique liées pour une grande part à la spécificité de ces objets. Il est commun pour les chercheure's qui travaillent sur le jeu de se confronter à la "barrière de trivialité ", selon l'expression de Sutton-Smith (1970), qui discrimine et confine au négligeable et à l'inutile les travaux sur des objets peu légitimes socialement. Pour aller dans le même sens, Brougère (1992, p. 10) ironise, à propos du jouet, en avançant qu'« une réflexion sur un objet futile ne peut être qu'une réflexion futile». Il en va de même pour l'animation. Coordonnant un des ouvrages les plus récents sur ce domaine, Camus et Lebon $(2015$, p. 9) l'introduisent par cette question directe : « L'animation peut-elle être digne d'un intérêt scientifique? " Ils notent que la "forme d'indignité sociale qui frappe l'animation [...] semble condamner toute initiative d'enquête, d'analyse, de publication... qui consacrerait du temps et de l'énergie à un objet futile. » A fortiori, on pourrait se demander s'il est bien nécessaire de consacrer un dossier à cette thématique. C'est en quelque sorte le défi de ce numéro et le pari de ce texte introductif que de montrer cet intérêt et comment le croisement de ces deux objets jugés triviaux permet de contribuer aux réflexions, plus générales, des sciences du jeu.

2 Avant de présenter les articles réunis dans le dossier, il nous parait important de cerner, dans un premier temps, comment le flou qui entoure l'animation participe de sa méconnaissance. Pour éviter l'écueil de nombreuses publications, nous proposons d'aborder le problème définitionnel de l'animation sous un autre angle, emprunté au jeu. Nous avons tenté de retracer la généalogie de cette notion pour le moins éclectique et polysémique à travers diverses disciplines. Dans un deuxième temps, nous revenons sur la rencontre du jeu et de l'animation qui, historiquement, s'est réalisée dans les patronages et les colonies de vacances les précurseurs de l'animation socioculturelle. 
Plus directement à partir de nos travaux, nous présentons plus longuement les relations ambivalentes entre le loisir et l'éducation, dont témoignent les usages du jeu, qui traversent l'animation et auparavant l'éducation populaire. Enfin, nous présentons les articles de ce dossier et comment, à leurs manières, chacun d'eux alimente les explorations précédentes selon des perspectives théoriques et méthodologiques multiples tout en conservant une focalisation sur l'animation auprès d'enfants et de jeunes dans un contexte de loisir.

\section{Problèmes définitionnels}

Outre son caractère indigne et trivial, l'animation partage avec le jeu un même écueil dès lors qu'on tente de circonscrire l'objet. Le travail d'élaboration du dossier confirme ce que les analyses ont déjà montré, à savoir que la tâche se révèle d'emblée difficile du fait du flou et des contours très larges de ces deux notions. Que ce soit le jeu ou l'animation, on est confronté aux mêmes résistances pour définir les termes - même si les raisons sont en partie différentes - puisque la multiplicité des usages du mot renvoie de fait à des réalités ou des représentations très hétérogènes qui n'ont souvent de commun qu'un "air de famille" (Wittgenstein, 2004 [1953]). Ces aspects de définition sont sensiblement éclairés concernant le jeu à la suite de la proposition de Brougère (2005, p. 53 sqq.) concernant "l'art de ne pas définir » et au travers de la revue Sciences du jeu qui vise, justement, à étudier la "malléabilité » et les variations des formes ludiques au sein des pratiques sociales. De façon symétrique, et pour répondre plus spécifiquement à la thématique de ce dossier, il paraît essentiel de prendre ici un moment pour préciser de quoi on parle en évoquant le terme d'animation.

\section{Une notion éclectique}

4 Pour ne donner que quelques exemples, le substantif (animation) ou le verbe (animer) apparaissent régulièrement dans des expressions comme animation commerciale, animation d'une émission radio ou de télévision, animer une formation, animation d'équipe, animation de soirée, animation de rue, animation de réunion, ou encore animation d'atelier. Le même terme désigne ainsi des pratiques sociales qui se situent sur de multiples registres tels que la promotion et la vente d'un produit, la présentation ou commentaire d'une émission plus ou moins conversationnelle, l'organisation ou scénarisation d'une activité d'apprentissage, la motivation et coordination d'individus pour agir et réfléchir ensemble, la mise en place d'une ambiance festive ou de divertissement ou encore l'orchestration ou dynamisation d'un échange ciblé sur un contenu. Cette diversité montre que le terme est associé à différentes dimensions qui sont autant de registres de sens. L'animation est pour partie une animation de groupe ou d'un "public», (animation d'équipe, animation de réunion, animation en gérontologie, animation d'une communauté internet, animation enfance-jeunesse, animation en insertion, etc.) pour partie une animation d'un espace particulier (animation de rue, animation en établissements culturels ou sanitaires, animation en camping, animation en magasin, etc.), l'animation d'un temps spécifique (animation de soirée, animation estivale, animation d'un festival, animation scolaire, animation d'un événement, etc.), l'animation d'une activité ou d'un atelier (animation théâtre, animation escalade, animation de formation, de conférence, etc.), ou encore 
l'animation d'un objet (animation d'une émission, animation de marionnettes, animation d'un cours, animation d'un support graphique pour un film ou dessin animé $^{1}$, etc.).

On peut ajouter que l'animation peut aussi être attachée à divers domaines ou champs d'action plus larges (animation culturelle, animation pédagogique, animation scientifique, animation sportive, animation sociale, animation touristique, animation politique, etc.) comme en attestent les différentes dénominations de diplômes. Ces registres explicitent et mettent à jour différentes dimensions sous-jacentes qui sont autant de caractérisations potentielles de l'animation, celle-ci se laissant difficilement saisir selon une seule de ses perspectives. En ce sens, l'étymologie du terme apparaît rapidement insuffisante pour recouvrir cette diversité, même si l'idée de « donner une âme $^{2}$ » sert parfois d'étendard ou de bannière éthique. Ainsi, en conservant le plus grand dénominateur commun à ces registres, on peut synthétiser en disant que l'animation renvoie - globalement - à des pratiques humaines et sociales qui supposent un groupe ou une dynamique relationnelle, un espace physique ou social, une temporalité (plus ou moins) distinctive, des supports matériels (plus ou moins) essentiels, éventuellement un attachement institutionnel et un ancrage thématique plus ou moins spécifique.

6 Pour compléter ce panorama, il faudrait poursuivre avec l'inventaire des expressions qui désignent les acteurs d'une part et les techniques et modalités d'autre part. Quels que soient les contextes et les usages, l'animation se comprend toujours comme une production, le résultat d'une élaboration plus ou moins anticipée ou improvisée (on fait une animation comme une performance artistique) et donc le fruit d'un - ou plusieurs agent (une personne réalise l'animation) qui en est en quelque sorte l'auteur et le réalisateur. Mais sur ce point, on peut voir l'extension des applications du terme puisque si celui d'«animateurrice» domine largement, c'est parfois d'autres désignations professionnelles qui apparaissent. Ainsi, il s'avère que d'autres métiers connexes, sans en avoir le titre, disposent d'une mission ou d'une fonction d'animation tels les enseignante's ou les formateur.rice's d'adultes pour l'animation de séquences pédagogiques, les présentateur.rice's de médias où sont animées des émissions ou un jeu télévisé, les intervenante:s du monde artistique et culturel... Pour le dire autrement, l'animation ne peut se résumer à la seule profession et à ce que recouvre ce titre, si tant est qu'elle puisse être elle-même définie.

\section{La généalogie d'un air de famille}

7 Si le sens commun se confronte quotidiennement aux implicites et aux confusions d'un terme polysémique, qu'en est-il du côté de la recherche et des travaux universitaires? L'étendue des contenus, contextes et formes d'action sous-jacents aux usages du terme d'animation est très vaste et difficile à embrasser dans son ensemble, y compris dans une approche sémantique (Carletti, 2014). A fortiori, une perspective de recherche globale n'aurait que peu de sens du fait de l'hétérogénéité des objets à considérer. Les recherches bibliographiques sur le sujet montrent toujours des entrées spécifiques et circonscrites à un domaine ou à un contexte de pratiques. Sans chercher et à défaut de pouvoir être exhaustif, on peut pointer des analyses issues de diverses disciplines (sciences de l'information et de la communication, sociologie, sciences de gestion, 
sciences de l'éducation ou psychologie sociale) qui s'intéressent, à leurs façons, au(x) métier(x) de l'animation.

8 Des analyses décortiquent ainsi le métier d'animateur télévision tant du côté de son évolution au sein du paysage ou système audiovisuel où les équilibres entre groupes sociaux - en l'occurrence entre animateur, directeur, producteur, réalisateur - varient au fil des restructurations institutionnelles, que du côté des innovations à partir desquelles se constitue son «art d'exercer» dans le secteur audiovisuel (Leroux \& Riutort, 2006) ou radiophonique (Méadel, 1984). Plus précisément, la profession se distingue et se valorise par ce "spectacle de la parole » qui, comme les décors, les éclairages, et les ponctuations musicales, participe de cette "grande mise scène" caractéristique de l'évolution des programmes (Chivot, 2004). On retrouve ici un des aspects à la fois de meneur et de fou du roi (joker) qui irrigue une partie des pratiques d'animation. Davantage dans une perspective d'analyse du travail, les postures professionnelles des animateurs de site en ligne ont été analysées pour mettre en évidence les tensions entre engagement dans la pratique et distance au rôle en particulier dans le cas de chat rose (Stoian, 2011). Apparaît là une partie de ce qui caractérise l'animation à la fois en tant que prestation qui est réalisée de façon plus ou moins impliquée par cet auteur de l'animation au sens de concepteurrice mais aussi réalisateur.rice, et dans ce rapport aux publics, spectateur.ricess devenant parfois participantees (Mehl, 1994), qui demeure un aspect essentiel de la pratique, notamment pour conserver leur attention, leur écoute et donc l'audience (Hennion \& Méadel, 1986).

9 Les animations relevant du monde commercial sont plus rarement étudiées. Elles renvoient au début des pratiques du libre service où le souci de mise en valeur de l'espace de vente, davantage que des produits, passait par des attractions comme des jeux, des tirages au sort ou des démonstrations de cirque (Grandclément, 2004). Les analyses de Clark et Pinch (1995) sur les camelots et les bonimenteurs semblent parmi les rares études qui se soient plus précisément penchées sur ce qui relève directement de l'activité des professionnelle's. Comme le souligne Grandclément (2004, p. 17), ils mettent en évidence les quatre étapes qui organisent leur pratique et les interactions : " attirer et construire une audience, décrire les biens et en manifester les qualités, constituer la valeur des biens et en donner un prix, obtenir un engagement de la part des clients potentiels et les transformer en acheteurs ». Autant d'éléments que l'on observe dans les « rebonds » orchestrés dans les conversations téléphoniques lors des appels pour une assistance technique analysées par Licoppe (2006). On retrouve ainsi, dans ce qui apparaît comme un savoir-faire ou un art, des traces des dimensions essentielles de l'animation: à la fois la captation (de l'attention) du public, l'explicitation et la promotion d'un contenu pour intéresser ou convaincre, et la finalité de créer un engagement dans une action. L'introduction dans les années 1990 des «aspects expérientiels» de la consommation introduit des recherches sur le «marketing expérientiel » et donc en partie sur « la fabrication de l'expérience de la part des entreprises » (Grandclément, 2004, p. 7). Les pratiques et les analyses sont posées en termes d'ambiance, d'immersion, d'« espace thématique dont la vocation est de contribuer à ce que les consommateurs découvrent un peu de rêve, s'évadent de leur quotidien" (Hetzel, 2002, p.9, cité par Grandclément, 2004, p.8). Les centres commerciaux [malls], mais aussi les «magasins étendard» (d'une marque) montrent la mise en place d'une offre d'activités débordant largement de la seule vente et « un travail d'animation de la flamme consommatrice chez le passant nonchalant.» 
(Grandclément, 2004, p. 16) Même si le terme animation n'est pas explicité, il s'agit de "ré-engager " et d'« impliquer ", autrement dit, d'une démarche à destination d'un public et qui vise à insuffler une dynamique par le truchement de moyens ici davantage matériels qu'humains.

Par ailleurs, l'animateur apparaît comme une figure phare - mais tout autant oubliée dans le champ de l'économie et de la gestion comme le montre un des rares articles qui lui est consacré (Remoussenard-Pourquier \& Ditter, 2015). Dans une approche en termes de management, l'analyse porte sur ce rôle clef de celui qui est chargé d'assurer la mise en œuvre et le développement d'un groupement partenarial d'entreprise (cluster). Le contexte est donc celui de la construction - à l'initiative des pouvoirs publics - d'un réseau d'acteurs économiques regroupés sur un territoire afin de passer d'une dynamique de concurrence à une dynamique de coopération permettant un développement économique individuel et collectif. On y retrouve les différents aspects qui traversent cette partie de l'animation focalisée sur la construction et la dynamisation d'un groupe. Il s'agit de fédérer un ensemble d'acteurs hétérogènes autour d'un projet qui tient lieu à la fois de ciment et d'objectif. Le travail passe par le repérage et la valorisation des compétences individuelles tout en trouvant les formes pour en faire émerger de la compétence collective qui va en retour permettre à chacun de s'inscrire dans une dynamique de changement. Les observations des pratiques révèlent l'importance de qualités d'écoute et relationnelle, mais aussi toute la diplomatie nécessaire pour parvenir à mettre en relation des personnes, caractéristiques qui traversent régulièrement le champ de l'animation.

11 Plus largement, le management puise dans les techniques d'animation de groupe pour conduire et animer des équipes. Hors des sciences de gestion, la psychologie sociale (de Visscher, 2011, 2014), propose des outils d'analyse de la dynamique des groupes (restreints) en poursuivant les travaux princeps de Lewin (1959). Sans oublier les propositions non-directives de Rogers (1966), ces «outils» et «techniques» ont largement été diffusés dans les années 1970 (Anzieu \& Martin, 1997), dans les milieux de la formation d'adultes et de l'animation socioculturelle (voir par exemple Limbos, 1971, 1974). Parenthèse historique, il convient de rappeler que les premières expérimentations de Lewin et ses collaborateurs sur la menée de groupe [leadership], le climat social et les comportements agressifs s'étaient déroulées dans les équivalents passés d'accueils périscolaires avec des groupes d'enfants réunis pour des activités manuelles (voir Lewin \& Lippitt, 1938 ; Lippitt \& White, 1943).

12 Par ailleurs, ces techniques d'animation et de gestion des groupes - parfois de la classe - se retrouvent également dans le domaine de l'enseignement. C'est particulièrement le cas des professeurs d'éducation physique et sportive dont les contenus d'enseignement se rapprochent voire se confondent en partie avec les pratiques de loisir et sportives des élèves. Bret (2008) décrit ainsi les liens particulièrement forts entre l'enseignement et l'animation chez ces professeurs dans les cursus de formation. On voit ici apparaître des proximités ou glissements entre les formes pédagogiques du monde de l'animation et $\mathrm{du}$ monde scolaire qui traversent une grande partie des pratiques sans être réellement interrogées ni par les professionnels ni par la recherche, comme développé plus loin.

13 Si ces quelques références ne peuvent évidemment couvrir l'ensemble des publications, la recherche bibliographique a cependant permis de constater le peu de travaux qui portent sur l'animation ou l'animat·eurrice. En dehors de ces entrées thématiques qui 
restent très peu développées, une partie importante des écrits relève en effet davantage des différents domaines professionnels pour en diffuser des méthodes, des techniques, des conseils en restant dans une perspective très pragmatique, voire prescriptive. Si, comme on peut l'entrevoir dans les quelques analyses repérées, des convergences existent qui auraient pu nourrir les contributions de ce numéro, force est de constater que le champ des pratiques qui touchent aux temps, activités, espaces ou institutions de loisir, est très nettement dominant. En ce sens, ce numéro est conforme à ce constat et reste centré sur ce domaine de l'animation de(s) loisir(s).

\section{La permanence des flous de la recherche}

Depuis les premiers travaux scientifiques qui s'intéressent à l'animation, et notamment ceux qui notent l'émergence et l'autonomisation d'un nouveau groupe professionnel dans les années 1960 (Besnard, 1972 ; Simonot, 1974), la définition de l'animation s'est heurtée à sa diversité de façon comparable aux tentatives de s'accorder sur une définition de l'éducation populaire (Besse, Chateigner \& Ihaddadène, 2017). Comme le résume Précas (1984, p. 113) à propos de la «littérature de l'animation», elle est « inépuisable » et " produi[t] des discours idéologiques favorisés par le flou qui entoure ce concept ». Moulinier (2008, p. 176) juge plus largement que « les termes d'animation, d'éducation populaire, d'action culturelle sont [...] semblables aux icebergs: non seulement ce que l'on voit recouvre une réalité souterraine considérable, mais en outre ce sont des notions fondantes, glissantes, nomadisantes, instables. » $\mathrm{Ce}$ «flou » persiste encore aujourd'hui pour Camus et Lebon $(2015$, p. 12), mais il est cette fois devenu l'objet d'une analyse afin d'appréhender les "mécanismes historiques et sociaux qui [le] produisent » dans la perspective de mieux comprendre les "différentes facettes » de l'animation. Cette section ne vise pas à l'éclaircir ni même à déployer l'« introuvable définition » (Gillet, 1995), mais plutôt à présenter les difficultés définitionnelles qu'ont aussi rencontrées les recherches précédentes pour cerner un objet protéiforme qui ne s'est pas stabilisé depuis. Encore récemment, Greffier (2013) explore, à défaut d'une définition toujours « difficile » $(\$ 2)$, ce « système » (\$ 9) nébuleux et un « labyrinthe de formations et de diplômes » (\$23).

L'animation peut ainsi référer à un groupe restreint et spécialisé de professionnellle·s et à un ensemble de pratiques comme pouvait le suggérer Poujol (1972) dans une première recension des recherches réalisées : «l'Animation, c'est ce que fait l'animateur et/ou, plus évasivement encore, "l'Animation", c'est ce que voudrait faire l'animateur ». Et pour éviter le caractère pour le moins tautologique de cette définition, l'auteure précise qu'il « resterait, alors, à définir l'animateur: l'animateur, pour nous, est celui qui se dit animateur à titre principal dans l'activité qu'il exerce». Cette restriction permet alors de réduire l'animation aux pratiques identifiées en tant que telles, qu'elles soient bénévoles, volontaires ou salariées. De nouveau, la difficulté de définition est reportée puisqu'il s'agit alors de délimiter les frontières - mouvantes - de ce groupe professionnel. En effet, portés par une institutionnalisation étatique, appuyés sur des diplômes rattachés à plusieurs ministères (affaires sociales, jeunesse et sport, enseignement supérieur), régulièrement enrichis par la création d'équipements (culturels, sociaux, éducatifs, sportifs, numériques plus récemment), de dispositifs et de politiques publiques visant des "publics» ou des «territoires", les métiers de l'animation présentent de plus en plus un caractère protéiforme à la croisée du travail social, de l'action culturelle, de la prévention et de la santé ou du monde scolaire. 
Plusieurs signes témoignent du développement, en même temps que de la dispersion de l'animation au fil des publications en France ; on peut évoquer la laborieuse estimation du nombre de professionnelle's concernée's (Gillet, 2006), la démultiplication des dénominations et titres d'emplois (les codes ROME [Répertoire Opérationnel des Métiers et des Emplois], la nomenclature Insee et autres définitions de poste), la variété et le renouvellement fréquent sur demandes ministérielles ${ }^{3}$ des filières de formation et des diplômes, la dispersion des ministères de rattachement, etc.

Comme le souligne Lebon (2009), les travaux et les publications de ces années 1970 s'intéressaient principalement aux fonctions et finalités de l'animation comme à la professionnalisation ou la reconnaissance de ses acteurs à travers l'étude de leurs profils et de leurs carrières. L'animation devient le sujet d'enquêtes de la part de l'Institut National (de la Jeunesse et) de l'Éducation Populaire (IN-J-EP) et quelques noms de chercheurs apparaissent régulièrement ${ }^{4}$. De la même façon que pour l'éducation populaire, dont elle serait une forme "professionnalisée " (Poujol, 1989, p. 33 ; voir aussi Labourie, 2008), les travaux et les recherches sur l'animation ont été largement réalisés par des personnes issues de ce monde ou dites « indigènes » (Lebon, 2008; Besse, Chateigner \& Ihaddadène, 2017) ${ }^{5}$. C'est peut-être une des raisons du caractère très global et souvent normatif - potentiellement idéalisé - des approches qui sont développées, formulant ainsi davantage ce qu'elle devrait être, qu'une réelle connaissance des pratiques effectives des animateur.rices. Pas ou peu de travaux s'intéressent aux pratiques animatives ${ }^{6}$ en tant que telles, comme le constatait déjà vingt ans plus tôt Précas $(1984$, p. $115 ; 2004)$ qui invitait à une «approche anthropologique » pour réduire la « suprématie » des « études quantitatives » oubliant, ou occultant, le quotidien des interventions. C'est un constat partagé par Besnard (1978) dans la rare note de synthèse portant sur l'animation où, face au problème définitionnel, il se propose de lister un ensemble d'éléments caractérisant les pratiques d'animation, quitte à établir un inventaire à la Prévert. Même s'il s'agit d'une " entreprise " jugée «laborieuse " (1980, p. 49) par l'auteur lui-même, elle conduit à une énumération d'une dizaine de traits (réponse à des intérêts, besoins, ouverte, en groupe...) dont la pertinence est toute relative puisque qui, pris un à un, ils ne sont pas exclusifs à l'animation et, pris dans leur ensemble, ils excluent des pratiques couramment dénommées « animation ».

Dans les années 1990 s'opère un tournant notable à partir des travaux de Gillet (1995) suite à sa thèse en sciences de l'éducation. Avec Augustin (2000), ils vont appeler au développement d'un « mouvement de recherche et d'expérimentation sur l'animation » selon le titre même d'un article (Augustin \& Gillet, 1997). Si ce mouvement a débuté au sein des colloques organisés à Bordeaux ${ }^{7}$ dès le début des années 1980 (Gillet, 2010), il va prendre une toute autre dimension au niveau international avec la création du Réseau International de l'Animation (RIA) et l'organisation régulière de congrès suivis par de nombreuses publications éditées et réunies dans une même collection. Malgré tout, Augustin et Gillet (2000, p. 152) reconnaissent que l'« évolution du champ de l'animation depuis plus de trente ans, sa progression continue et multiforme, ses succès et sa fragilité rendent aléatoire la recherche de définitions fiables et admises par tous ». Plus récemment, le dernier rapport de l'observatoire des métiers de l'animation (Segrestan \& Gouju, 2013, p. 7) constate même qu'il n'existe " pas de consensus au sujet d'une définition de l'animation ». De fait, malgré les tentatives récentes, dont celles de della Croce, Libois et Mawad (2011) ou de Lafortune et al. (2010), c'est le travail de Gillet (1995) qui reste le plus cité et semble établir un consensus, sans doute parce qu'il 
dépasse la recherche d'une définition. À la place, il propose une modélisation, basée sur une approche psychosociale, qui permet d'approcher une variété de pratiques animatives, selon qu'elles se dirigent vers un pôle «militant » (fonction d'élucidation), «technicien» (fonction de production) ou de «médiation» (fonction d'élucidation). Évitant une longue énumération, la combinaison des trois fonctions et des trois pôles ${ }^{8}$ permet de rendre compte et d'embrasser une complexité de pratiques qui peuvent évoluer et s'adapter selon les situations rencontrées (Gillet, 2008).

\section{L'ouverture complexe à d'autres pays}

Comme l'explique Gillet (2005a), le terme d'«analogie » est le plus adapté pour tenter de cerner des pratiques sociales proches, similaires, mais distinctes à l'animation française. En matière de comparaison internationale c'est aussi ce travail qui continue à faire référence faute de recherche de plus grande envergure. Après quelques anecdotes, l'auteur présente plusieurs définitions issues de travaux de différents pays et en particulier l'Argentine, l'Uruguay, le Brésil, l'Italie, l'Espagne, et la Finlande, en les liant aux traditions historiques et philosophiques de chacun des pays, et à leurs évolutions sociales et culturelles, et à leurs développements économiques et institutionnels. Il montre ainsi une forme d'unité autour de l'idéologie de l'éducation populaire portée par des valeurs démocratiques et républicaines qui s'est diffusée depuis la France vers d'autres pays européens dont l'Espagne et le Portugal avant de se frayer un chemin vers l'Amérique latine. Cette unité n'empêche pas une diversité due aux hybridations lorsque des idéologies et des théories (théologie de la libération, pédagogie des opprimée's, approche ethnocommunautaire, citées par Gillet) se rencontrent. La diversité des appellations qui ne se recouvrent que partiellement en témoigne déjà. Entre la pédagogie sociale [socialpädagogik] allemande ou suisse, le développement communautaire [community organizing] en Amérique du Nord, la récréologie au Québec, l'éducation sociale [educacion social], la recréation du temps libre [recreación del tiempo libre] ou le loisir [el ocio] dans les pays hispanophones, voire le travail de jeunesse [youthwork] en Angleterre notamment, il existe une variété de pratiques et de projets sous-jacents.

Dans chaque pays ou aire culturelle, l'animation et les métiers correspondants traversent plusieurs domaines en fonction de leurs rattachements institutionnels (éducation, culture, affaires sociales, loisir, sport...). Gillet (2005b, p. 20) peut ainsi conclure : "Dans le champ sémantique de l'animation se profilent, autour ou à côté de cette notion, des filiations, des cousinages, des hybridations, des métissages, des glissements, des mutations ». Cependant, sous l'égide de collaborations européennes, le travail de jeunesse [youthwork] et l'éducation non formelle sont deux terminologies malgré leurs limites - qui tendent à s'imposer dans les politiques et dispositifs européens, au risque d'une réduction de l'animation à une seule de ses facettes (voir Oberti, 2008 ; Berger, Labadie \& Wittman, 2018).

Derrière ces terminologies se tissent bien sûr des divergences entre pays, mais les analyses montrent aussi la diffusion transnationale des mêmes tensions et courants. Sans pouvoir rentrer dans le détail de ce que recouvre le domaine pour aire culturelle, on peut en comprendre la complexité sur un exemple européen. Situés au carrefour de l'aire francophone et germanophone, les analystes suisses comme Moser et al. (2004) 
sont particulièrement bien placés pour éclairer ces passages de frontières récurrents depuis l'émergence du domaine et des théorisations sur la notion.

21 Les auteurs constatent qu'« en Allemagne, depuis ses origines le champ de l'animation a été rangé dans les sciences récréatives et la pédagogie des loisirs » (Moser et al., 2004, chap. 1 , § 14) et non dans un registre plus largement social et/ou culturel comme en France. La comparaison internationale permet ainsi de faire apparaître deux visions dominantes. L'une privilégie la dimension sociopolitique et conçoit l'animation dans une optique de changement de la société en aidant plus particulièrement les individus en difficultés sociales à trouver leur place. L'autre privilégie l'individu et vise à faire un usage judicieux des loisirs qui se sont développés avec le temps libre et qui sont perçus à la fois comme des potentiels et des dangers. Dans ce schéma, la France se situe davantage dans l'approche plus sociopolitique du fait du poids de l'éducation populaire qui a mis en avant le partage et la justice. À l'inverse, l'Allemagne se situe plus du côté $\mathrm{du}$ loisir, en partie en réaction à l'influence du régime national-socialiste sur les mouvements de jeunesse qui a retardé les ouvertures qui se sont développées en France. Mais les analyses montrent par ailleurs les tensions entre ces deux approches au sein même de chacun de pays. En France, la mise en exergue de la Culture dans les années 1960 sous l'impulsion de Malraux ouvre la voie vers un domaine hybride souvent désigné de «socioculturel». Le développement des accueils de vacances pour enfants peut être perçu comme un autre élément qui oriente l'animation française davantage vers le loisir que vers le sociopolitique. En Allemagne, l'apparition du domaine de la "pédagogie sociale", mais aussi du "socioculturel» montre une réorientation vers l'action sociale. Mais "le fossé entre la pédagogie des loisirs et la mission des centres socioculturels était trop profond (chap. 2, § 28) pour se retrouver sous une appellation commune. "Le terme générique Pédagogie des loisirs/sciences des loisirs [...] semble avoir alors absorbé celui d'animation, laquelle n'apparaîtra plus qu'en relation avec l'offre de loisirs destinés aux enfants » (chap. 2, § 27). On peut souligner ici que la dénomination allemande met en exergue la tension qui traverse les frontières du monde de l'animation et qui apparaît dans les pratiques et les courants allemands entre les tenants de l'éducatif et ceux qui s'en défendent pour valoriser les dimensions de loisir des accueils.

Dans ce contexte, la Suisse devient ainsi le terrain de rencontre de l'opposition entre la pédagogie des loisirs allemande et le mouvement de l'animation francophone qui apparaît spécifique par son ancrage dans l'action sociale et vers le changement social (Moser et al., 2004). Si la Suisse romane a rapidement adopté l'orientation française avec toutes ses tensions, la Suisse alémanique semble davantage prise entre les deux orientations. Curieusement, le terme animation se présente comme une solution puisqu'une partie des acteurs du secteur le reprend pour se démarquer à la fois du travail communautaire et ne pas apparaitre comme des travailleurs sociaux, et de la pédagogie des loisirs pour ne pas être perçus comme des pédagogues, mais davantage comme des soutiens à l'initiative personnelle et à l'organisation collective (Ibid.).

Pour finir sur ces aspects internationaux, il faut souligner que ces diversités de perspective, de délimitation ou d'appartenance de champ, se retrouvent ou ressurgissent systématiquement lorsqu'il s'agit de les traduire d'une langue à l'autre. Le Réseau International de l'Animation est depuis de nombreuses années confronté à ce problème : «le caractère intraduisible en anglais et en allemand de ces deux mots : animation et animateur. Il ne peut s'agir d'une lacune des langues concernées, mais 
bien de notions idéologiques non transportables d'une société à une autre. » (Poujol, 1989, p. 36). Ces mêmes difficultés ont été rencontrées pour l'écriture de ce dossier. Outre le terme "animation", les appellations des types d'accueils ne sont pas non plus transposables simplement du fait des organisation et structuration différentes selon les pays. La complexité française apparait ici autour du terme "accueil périscolaire » qui doit être distingué d'" accueil de loisir ", " centre de loisirs ", "centre de vacances", mais aussi "parc de loisir», etc. Pour entrer davantage dans les contenus ou les caractéristiques de l'animation, on peut remarquer la difficulté de traduction d'un terme comme celui d'« encadrement » des enfants ou des activités qui apparaît comme une spécificité de l'« animation à la française " mise en évidence là encore dans une comparaison internationale comme on le verra plus loin. C'est bien sûr lors de la traduction du texte anglais que ces difficultés ont été les plus lisibles; par exemple autour de terme comme playleader ou playleadership ou encore pour traduire la distinction entre waste material playground et junk playground, d'autant plus quand ces termes sont déjà eux-mêmes des traductions du danois. De plus, même les traductions qui ne semblent pas poser de problème révèlent souvent à y regarder de près des distorsions qui ne sont pas sans conséquence. C'est notamment le cas d'adventure playground qui est généralement traduit par «terrain d'aventure» pour ne pas confondre avec l'expression «terrain de jeu » qui désigne plus largement des espaces pour jouer. Or, cette traduction fait disparaitre la notion de jeu au profit de l'aventure ce qui est encore renforcée avec la traduction « terrain pour l'aventure ». Par ailleurs, comment traduire le terme de playground en français pour tenir compte de la distinction entre le singulier (du jeu) ou le pluriel (des jeux). Évidemment, le même problème intervient avec le vocabulaire spécifique à la tradition typiquement anglaise du playwork présentée dans un article du dossier qui s'est avéré difficile, voire impossible à traduire en français. Pour le moins antinomique, l'expression de «travail du jeu » ou de «travail ludique » - et ses «travailleurrse's » - reflète difficilement les pratiques concernées et paraît trop en contradiction avec les caractéristiques du jeu. Paradoxalement, le texte révèle que des pionniers de ce mouvement auraient préféré la dénomination (française) d'« animation », qui a été abandonnée à défaut d'en trouver une traduction satisfaisante en anglais.

En somme, qu'elle soit abordée comme un champ, un secteur ou un domaine, l'animation ne peut être cernée ni par le métier d'animateur ou plutôt les métiers de l'animation tant ceux-ci sont variés et surtout peu structurés (Lebon, 2009), ni par les institutions qui recouvrent un large panorama d'organisations et de structures qui se positionnent sur le temps libre entre l'école et la vie familiale, ni par le domaine d'action qui est couramment situé au carrefour de l'éducatif, du social et du culturel. L'animation apparaît ainsi comme un «monde social» (Becker, 1988) qui se construit par ses acteurs et ses actrices, ses publics, ses organisations, ses politiques, mais aussi ses rites et peut-être ses mythes, dont celui du jeu. Mais, la construction reste difficilement saisissable par ses contenus, ses actions, ses pratiques tant elle est étendue, et diversifiée et mal identifiée y compris par les acteurs de ce monde euxmêmes. Ce qui conduit à penser que « le label "animation" est peut-être le seul principe d'unification" (Mauger, 2015, p. 160). Comme certains le formulent en suivant une perspective «nominaliste» (Lebon \& de Lescure, 2016) à propos de l'éducation populaire - ce domaine connexe et parfois confondu dans le contexte français -, l'animation "ne peut se définir que comme l'ensemble "des choses" qui utilisent "le 
mot" pour se définir » (p. 165). Or, il s'avère que le terme de loisir(s) est souvent associé à l'animation et pourrait permettre de mieux cerner les flous qui l'entourent.

\section{Le(s) loisir(s) : un centre de gravité historique}

25 Au-delà de ce consensus sur une « identité instable » et " faiblement institué » (Lebon, 2009, p. 4) de l'animation, les publications sur le domaine se retrouvent pour souligner une autre dimension, celle du loisir. Avec les bouleversements des temps sociaux du $20^{\mathrm{e}}$ siècle, liés à l'évolution de la société industrielle et à la réduction du temps de travail rémunéré, l'animation est très régulièrement associée à la "civilisation des loisirs » annoncée par Dumazedier (1962) et inscrite dans la «société du temps libre » mise en exergue par Sue (1982). Ainsi, l'animation peut être définie, par des chercheures, comme le métier qui « agit dans et sur le loisir des autres » (Poujol, 1989, p. 78) ou qui «travaille dans et sur les temps libres des autres » (Bordes, 2007, p. 102). À mesure que le temps libéré des obligations salariales s'est accru (législation de la durée hebdomadaire du travail, semaines de congés payés, repos, réductions diverses, etc.), le loisir et les pratiques culturelles ont aussi pris leur essor pour combler la vacance de ce nouveau temps social (Sue, 1988), soutenus par la planification et la construction de milliers d'équipements sportifs et socioculturels (Augustin \& Ion, 2017). Or, pour assurer leur déroulement dans un équipement, pour gérer et organiser leurs mises en place, pour accueillir, accompagner et encadrer les personnes, ces pratiques se sont largement appuyées sur le monde associatif - en partie déjà installé par l'éducation populaire - d'où a peu à peu émergé le groupe des animateurs et les animatrices et les premières formations dès les années 1960 (Poujol, 1989).

L'animation se développe dans les différents secteurs entre lesquels le loisir se décline : aux loisirs culturels correspond l'animation culturelle et ses institutions; les activités sportives disposent de leurs équipements et de leurs professionnelless; les pratiques artistiques composent avec leurs spécialistes, sans oublier le monde du tourisme, celui du divertissement et des médias, etc. On pourrait ainsi continuer à l'envi pour souligner que, quel que soit le public impliqué (des mineurees, des adultes, des personnes âgées ou des personnes ayant des besoins spécifiques) ou le territoire considéré (urbain, rural...) ou encore le domaine de pratiques, les multiples activités qui trouvent leur place dans le loisir ou dans le temps libre sont traversées par ce monde social qu'est l'animation, peuplé par des animatrices et des animateurs dont les spécialisations varient et se combinent. Ces divers.es professionnelle's peuvent aussi intervenir et organiser des activités de loisir dans des institutions détachées du loisir. Ponctuellement ou de façon pérenne, des animatrices et des animateurs sont recrutés dans des établissements où résident des personnes dépendantes ou atteintes de handicaps à côté ou à la marge des soins (Lebon \& Lima, 2011), dans des hôpitaux notamment pour les enfants (Pombet, 2014), dans des Instituts Médico-Éducatifs ou un Centres Éducatifs Renforcés en plus de l'orientation disciplinaire et rééducative ou dans des équipements culturels (bibliothèques, médiathèques, etc.) ou encore, dans des Foyers de Jeunes Travailleurs (FJT) plus proches du monde du travail et de la formation d'adultes. En un sens, le flou qui entoure le loisir et ses multiples pratiques se confond avec le flou de l'animation et ses différentes orientations.

Ne pouvant se définir uniquement comme des pratiques sociales - au sens " des loisirs » - ni comme une quantité de temps - au sens «d'un temps libéré»-, le loisir est 
dépendant des personnes qui s'y adonnent et de leurs relations à ces pratiques (Sue, 1988 ; Yonnet, 1999). En conséquence, l'animation ne peut être un "système constitué " ou stable, et restera un "système en voie de constitution" (Besnard, 1985, p. 66) permanente aux grès des évolutions du loisir". En prenant l'exemple des «enfants qui jouent entre eux sans animateurs » ou des "adultes qui se réunissent pour passer la soirée ensemble ", Besnard (1985, p. 43) va jusqu'à évoquer une " animation naturelle » à côté d'une « animation institutionnalisée » dont l'existence est jugée « essentielle ». Si l'intérêt de cette approche totalisante est limité - si tout devient animation, rien ne l'est - elle permet d'en souligner le caractère intrinsèquement mouvant et fluide, en relation avec les pratiques de loisir des personnes concernées. Autrement dit, si le loisir est difficile à circonscrire, l'animation le devient tout autant. Cette concomitance entre loisir et animation fait apparaitre un facteur essentiel de l'instabilité de l'animation. La difficulté à saisir le loisir ne peut que renforcer la difficulté à appréhender l'animation. D'une part parce que l'extension et la démultiplication des loisirs offrent autant de prises à l'animation en même temps qu'elles en provoquent l'éclatement. D'autre part parce que, si tant est que l'organisation des loisirs pouvait conduire à organiser l'animation, il resterait néanmoins un flou attenant au caractère personnel et subjectif du loisir. Une partie de ce qui occupe le temps libre reste ainsi loisir sans forcément qu'il y ait d'animation, sauf à suivre l'approche extensive de Besnard (1985).

Cependant, plusieurs auteurs estiment insuffisant et dommageable ce rapprochement de l'animation au loisir, même si c'est toujours subtilement évoqué. Pour Besnard (1980, p. 22), «l'Animation [...] ne saurait se limiter au secteur du temps libre », tout en reconnaissant qu'il s'agit de «son secteur dominant». Moulinier (1978) admet aussi que « l'occupation des loisirs » est le « secteur » socioprofessionnel de l'animation, mais on ne peut «limiter à ce secteur la population des animateurs » vu l'étendue des secteurs où ils interviennent. Si la fonction sociale "récréologique » est reconnue, Besnard (1980, p. 33) évoque ses craintes que l'animation s'éloigne « de la conception de l'éducation populaire " pour se rapprocher des "perspectives récréologiques » du " récréationnisme américain avec une armée de techniciens du loisir ». De façon moins tranchée, dans une comparaison avec la situation québécoise où la récréologie est la discipline principale des formations universitaires, Poujol (1989, p. 48) interroge « Quel animateur français accepterait que les sciences du loisir soient les plus appropriées à théoriser son activité ?» Plus loin, elle souligne que les «animateurs n'aiment pas qu'on évoque leur action en termes de loisir et d'occupation du temps loisir » (p. 60) en écartant la figure stéréotypée de l'amuseur ou du "clown ». Derrière ces assertions, souvent implicites, apparait une distanciation - plus ou moins prononcée - par rapport à la frivolité du divertissement rattachée au loisir. Cette prudence vis-à-vis du loisir se dégage aussi des approches et définitions de l'animation qui lui attribue une dimension éducative et morale - par l'entremise de «bons » loisirs, légitimés par l'encadrement assuré par des professionnelle's - voire une portée sociale et politique, incluant une transformation de la société. Le loisir ne saurait suffire à l'animation si l'on en croit Poujol (1989, p. 201) pour qui l'animation en tant qu'« héritière de l'éducation populaire, s'est sans doute détournée de l'école au bon moment", mais tout en se construisant sur le " rejet de toute idée de "loisir" ".

Des recherches plus récentes révèlent la pérennité de cette distanciation, voire de ce refus, du loisir pour une très large partie des professionnelle's qui accueillent les enfants dans les structures hors-scolaires, contribuant ainsi à laisser la notion largement impensée en particulier en ce qui concerne le public des enfants (Roucous \& 
Adam, 2016). Pourtant, l'animation doit beaucoup au loisir, plus précisément à la structuration des temps libérés et à l'organisation des loisirs de l'enfance qui va ouvrir la voie vers le développement du loisir des adultes. Car c'est bien l'obligation scolaire et son « pendant » que constituent les congés scolaires qui introduisent la nécessité d'une prise en charge des enfants hors de l'école. Les patronages et les garderies, ces "œuvres post-scolaires" (Lebon, 2005; Dessertine \& Maradan, 2001) qui se développent dès la fin du $19^{\mathrm{e}}$ siècle ne cesseront de s'étendre au fil du $20^{\mathrm{e}}$ siècle sous le double effet de l'allongement de la scolarisation obligatoire et du souci de contrôle et de sécurisation des enfants - soi-disant - livrés à eux-mêmes du fait de la généralisation de l'activité professionnelle des deux parents (Renaut, 2002). Pour Labourie (1978) ce développement des activités organisées en dehors de l'institution scolaire explique les prémices de l'animation à cette époque et son institutionnalisation progressive.

30 Avant de rentrer dans plus de détails sur ce lien entre animation, loisir, enfance et jeu, il s'agit de souligner que le loisir est aussi un monde traversé par le jeu et l'animation. Parallèlement à l'articulation entre loisir et animation développée précédemment, Brougère démontre un lien essentiel entre loisir et jeu. À partir des analyses d'Elias et Dunning (1994) et de Yonnet (1999) de la forme sociale du loisir et de ses caractéristiques, l'auteur relève les nombreux recouvrements avec la forme ludique et les critères retenus et il peut affirmer que le jeu « est une forme de loisir » (Brougère, 2005, p. 121), voire sa première expression. Cette proximité pourrait laisser penser qu'en tant que métier du loisir, l'animation deviendrait, en quelque sorte, un métier du jeu. En se penchant plus précisément sur ce rapprochement entre jeu et animation dans la prochaine section, on constatera que l'articulation est loin d'être évidente.

\section{Un croisement à explorer}

Si, comme on l'a évoqué précédemment, les déclinaisons de la notion animation sont nombreuses, dans chaque domaine le jeu apparaît très vite et très régulièrement comme un des objets ou un support de l'animation : animation d'un groupe de rôlistes, animation d'un club de jeu d'échec, animation d'une ludothèque, animation d'un festival de jeu, animation d'un jeu de société, ou encore animation d'un jeu télévisé ou radiophonique, animation d'une boutique de jeu, animation par le jeu dans la formation des adultes. On retrouve ainsi du jeu à tous les niveaux d'exercice de l'animation : du groupe (de joueurs), à l'espace (de jeu), en passant par le temps (de jeu), l'atelier ou club (de jeux), et surtout toute la variété des différents types de jeux (de société, de rôle, traditionnels, etc.).

Mais, les liens entre jeu et animation dépassent très largement ces articulations directes où le jeu est central quelle que soit la dimension par laquelle il est abordé. De fait, il suffit de porter son attention sur le monde de l'animation dans son ensemble pour saisir des liens qui apparaissent comme des allants de soi. Le jeu apparaît comme une évidence, voire une nécessité pour de nombreuses personnes, actrices ou observatrices du secteur, dès lors qu'animer est renvoyé à son sens étymologique de «donner vie ». En ce sens, c'est à travers ou par le jeu qu'une activité ou qu'un projet deviendra attractif et captivera les participants. "Rendre ludique» semble bien souvent devenir un synonyme d'animer. 
ependant, il apparaît rapidement que l'association ne peut être aussi tangible et rapide entre jeu et animation. Avant toute analyse précise, on comprend que les multiples dimensions des activités développées dans les différents espaces et temps de l'animation - qu'elles soient artistiques, sportives, manuelles, numériques ou autre -, dépassent mais tout en concurrençant la seule dimension de jeu. De fait, la tradition consiste à chercher à les faire cohabiter en faisant fi des tensions éventuellement perçues. Mais pour qui s'intéresse au jeu, ces évidences et ces allants de soi apparaissent vite empreints de déformations, de détournements, voire de méprises. À y regarder de plus près, les relations entre jeu et animation se révèlent prises dans une complexité que ce numéro souhaite interroger et éclairer.

\section{Une centration sur l'éducation de l'enfance}

Les liens entre jeu et animation semblent trouver leur origine dès l'émergence de cette dernière avec l'apparition et le développement des institutions fondatrices d'une partie $\mathrm{du}$ monde de l'animation que sont les colonies de vacances et les patronages. Les analyses historiques permettent de comprendre que le fondement se situe dans cet adressage de l'animation aux enfants et le prolongement de leur éducation, mais " antiscolaire» (Poujol, 1989; Lebon, 2005). Dans ces patronages, de nombreuses « activités » sont développées pour leur « puissance de séduction » telles le cinéma par exemple (Dessertine \& Maradan, 1998, p. 124). Dans cette perspective, le «jeu est souvent le premier argument donné par les curés et vicaires pour justifier l'intérêt de leur patronage » (ibid.). Rapidement, les organisations et leurs manuels à destination des monitrices et des moniteurs ${ }^{10}$ promeuvent l'usage du jeu (voir par exemple Guillen, 1942 ; Courtois, 1936 ; Schmitt \& Boulogne, 1955). Comme le souligne Lee Downs (2009, p. 205) « le mot "jeu" ne signifiait pas la même chose pour tous, mais tous partageaient la conviction que le jeu dévoile de façon naturelle les forces et faiblesses morales, les espoirs et les peurs les plus profonds de l'enfant ».

Qu'il s'agisse de préserver les valeurs chrétiennes ou républicaines et laïques ou de s'intéresser plus directement à la (bonne) santé des enfants, le souci de former les futurs membres de la société s'avère être le premier moteur de toutes les pratiques dès les débuts de ces institutions. La perpétuation de l'action éducatrice, apparue avec la mise en place de la scolarité, se réalise aussi à travers les colonies de vacances, portées par des considérations sanitaires et sociales, parfois politiques, puis uniquement pédagogiques, «neutralisées» par la puissance publique (Lebon, 2005; Lee Downs, 2011 ; Houssaye, 1989). Même si ces nouvelles instances trouvent leur place sur les temps libérés par l'obligation scolaire, elles sont tout autant voire davantage focalisées sur leur mission d'assistance et de protection - physique et morale - de l'enfance et de la jeunesse.

Cependant, au fil des décennies, les mouvements d'éducation populaire, et plus directement les patronages puis les centres aérés ou les colonies de vacances, sont pris dans un mouvement pour se détacher des pratiques scolaires tout en poursuivant des objectifs de formation de la jeunesse plus ou moins dictés, ou contrôlés, par l'État selon les périodes. Mise au ban de l'École, l'éducation nouvelle et les méthodes de pédagogie active investissent le loisir et prennent davantage de place juste avant la Deuxième Guerre mondiale, même si elles se trouvent très rapidement confrontées à une forme d'organisation et de normalisation portée par l'institutionnalisation du domaine avec la 
création de diverses instances et agréments. En résumé, Houssaye (1998, p. 104) considère que « le centre de vacances est et se veut une école (nouvelle, active, idéale) et un lieu éducatif total et idéal. » Proches de la nature et éloignés d'un urbanisme vicié par les maladies, les pédagogues peuvent modeler et maîtriser un milieu éducatif selon leurs convenances.

Mais avec plus de distance par rapport aux pratiques et aux idéaux, les recherches des années 1990 convergent pour mettre en évidence la proximité des modalités d'accueil des enfants dans les centres de vacances - ou colonies de vacances - avec « la forme scolaire » (Vincent, 1980). " Progressivement, le mode scolaire de socialisation, c'est-àdire la socialisation pensée et pratiquée comme "éducation", "pédagogie", etc., s'est imposée comme référence (non consciente), comme mode de socialisation allant de soi, légitime, dominant » (Thin, 1994, p. 43). Plus précisément, pour Houssaye (1998, p. 101) « le loisir en tant que tel n'est pas intéressant, c'est la forme éducative qu'il permet et qui le met en œuvre qui elle est recherchée et considérée ». La nature et les besoins de l'enfant - dont un besoin d'ordre - sont posés comme des fondements des pratiques éducatives qui cherchent à compenser les déficiences annoncées de l'institution scolaire mais aussi celles de la famille. C'est dans ce contexte historique d'émergence des accueils enfance et jeunesse que la question du jeu doit être abordée pour être saisie dans sa complexité.

\section{Formes ludiques au piège de l'éducation}

Dès les prémices des accueils, le jeu trouve sa place sur la base de cette conception romantique qui en fait une activité « naturelle » de l'enfant conduisant, si ce n'est à le valoriser, tout au moins à l'inscrire largement dans les pratiques. Cette approche «naturaliste» renforce les liens avec le monde de l'animation en pointant cette opposition à l'école et donc au sérieux qui a été un élément structurant des actions et des institutions hors scolaires. Le jeu spontanément associé au domaine de l'animation lui permettrait de se démarquer de la forme scolaire dans un mouvement assez similaire à ce qui a pu caractériser le préscolaire (Brougère, 1995). La comparaison peut être poursuivie pour souligner le même mouvement de déqualification ou de formalisation qui se dissimule derrière cette prévalence du jeu. L'usage des mots est à nouveau ici très révélateur puisque le jeu apparaît très régulièrement sous le terme de « ludique » pour caractériser toutes les pratiques et les instances des accueils : activité manuelle ludique, thématique ludique, sortie ludique, projet ludique, et bien sûr animation ludique. Le jeu se présente ainsi comme une - simple - qualité, " ludique ", qui est accolée, plus ou moins intentionnellement d'ailleurs, à de nombreuses pratiques de loisir. En d'autres termes, il est mis au service d'une autre pratique bien souvent sans aucun questionnement sur la réalité du jeu développé, ni sur une potentielle valeur de la dimension propre de l'activité. Roucous (2010, p. 205) constate très clairement que « le jeu apparaît souvent [...] comme une mise en forme utilisée par les animateurs pour donner un aspect ludique à tout ou partie des activités développées », en particulier dans les accueils qui valorisent les "activités " (manuelles, sportives, culturelles...) de façon dissociée des jeux. Ceux-ci sont alors une manière d'attirer et de séduire les enfants vis-à-vis des propositions des animateurs en leur donnant une «coloration ludique» (p. 213-214). Peu importe alors que la pratique soit parfois très loin des caractéristiques du jeu. 

l'éducation nouvelle dès les années 1930 et à son fondement sur «les besoins de l'enfant» qui s'appuie sur le développement et la légitimité grandissante de la psychologie de l'enfant (Ottavi, 2001). Comme l'analyse Houssaye, (1998, p. 102), "l'idéologie est désormais celle de l'enfance et de ses besoins, vus et réalisés par et dans l'Éducation nouvelle ». Les approches psychologiques de l'époque renforcent cet intérêt par l'affirmation du(des) «besoin(s) de jeu » et de leur évidence naturalisée mais avec toute l'ambivalence associée à cette notion (Burman, 2008). Le jeu se présente alors comme une nécessité quasi vitale et donc incontournable des pratiques d'animation dès lors qu'il s'agit d'enfants, tout en apparaissant simultanément comme une compensation dans la confrontation à d'autres besoins qui peuvent varier selon les périodes (repos, hygiène, sécurité, etc.).

doxalement, afficher le jeu comme un besoin conduit souvent à lui conférer un rôle secondaire par rapport à d'autres exigences jugées prioritaires. Parmi celles-ci le «besoin d'ordre présenté comme une nécessité intérieure pour l'enfant qui en tire satisfaction" (Houssaye, 1998, p. 102) est particulièrement problématique parce que antinomique avec ce qui caractérise le jeu. De fait, l'ordre se traduit dans les faits par la construction de multiples cadres qui organisent mais aussi délimitent les espaces, les temps et les activités, autant de mises en forme des pratiques qui visent à cet «encadrement» mis en exergue par Gram (2003). Dans une comparaison entre les Pays-Bas, la France et l'Allemagne, elle montre que même si les pratiques de loisirs s'avèrent largement «similaires» (p. 128) du point de vue des familles, la France se distingue nettement par l'importance accordée à cette notion d'encadrement qui apparaît d'autant plus forte au regard de la valorisation de l'autonomie qui domine aux Pays-Bas et en Allemagne. Dans ce contexte typiquement français, contrairement à ce que laissait supposer l'approche naturaliste, le jeu n'échappe pas au mouvement, et la «pédagogie du loisir » de l'animation va chercher à garder la main - et le pouvoir - en généralisant un «jeu dirigé ». Prévoir, organiser, circonscrire, mener, arbitrer le jeu vise à le faire rentrer dans un cadre d'expérience singulier. Il ne s'agit plus d'une modalisation - un cadre secondaire - créée par les participante-s pour donner un sens second à une situation (Goffman, 1991), mais un cadre fabriqué par les animat·eur.rice·s pour tracer les limites, les règles, les normes voire les interdits du jeu. Il est en réalité question de re-cadrer le jeu autrement dit de faire rentrer le cadre du jeu dans le cadre de l'animation.

41 En filigrane de ce court historique, cet encadrement du jeu est bien évidemment à lire au filtre de la question éducative qui traverse les accueils de loisirs depuis leurs origines. En parallèle et en contradiction avec l'idée d'un jeu naturel, une approche utilitariste voire instrumentale se dessine de plus en plus précisément au fil des décennies. Reprenant les conceptions de Claparède (1920, p. 429 sqq.) à propos d'une école sur mesure, le jeu est posé plus directement en termes d'outil et de moyen indispensables de l'éducation. La similitude avec les évolutions du préscolaire et plus particulièrement de l'école maternelle ne fait ici aucun doute. La "ruse pédagogique " telle qu'analysée par Brougère (1995, p.64), devient la norme jusqu'en dehors de l'institution scolaire malgré toutes les tentatives de l'animation pour s'en éloigner. Cette utilisation du jeu à des fins pédagogiques apparaît comme une évidence pour les acteurs du champ professionnel tant elle répond à cette perspective éducative qui s'impose quand on s'adresse aux enfants. On peut ainsi identifier une partie des 
accueils proches du «modèle scout " qui fait la part belle aux jeux tant qu'ils sont organisés, préparés, encadrés et qu'ils répondent aux objectifs et aux projets déterminés (Roucous, 2010, p. 221). Cette " pédagogisation » du jeu se traduit dans une mise en forme spécifique, une "forme ludique animative " observée dans plusieurs accueils et séjours (Besse-Patin, 2019). Celle-ci se distingue par la définition précise d'une temporalité, d'un espace délimité, de règles ou des consignes, d'un groupe fixe d'enfants, un matériel restreint voire des objectifs au-delà de la pratique du jeu et leur évaluation. En se penchant sur un ensemble plus large de types d'accueil, on saisit à quel point cette posture utilitariste traverse l'ensemble du hors scolaire de façon graduée entre « apprendre en jouant » qui laisse supposer une priorité au jeu et « jouer en apprenant » où le jeu est annoncé comme une dimension secondaire, même s'il est recherché parce que attrayant (Roucous \& Adam, 2016).

Il faut entrer par la question du jeu pour mesurer les effets de cette forme animative quant à la nature des activités ludiques - ou ludicisées - qui dominent les accueils de loisir. Que devient le jeu dans ce recadrage de l'animation? Comme le constate Houssaye (1998, p. 104) «le jeu est devenu tellement "actif" (en étant intégré "aux activités") et encadré qu'il perd sa nature, enfoui qu'il est dans la volonté éducative de l'encadrement ». Pour reprendre les analyses en termes de formes que propose Brougère (2010), l'observation attentive des pratiques montre les diverses déform(lis)ations que traverse la forme ludique originale du fait des intentions éducatives des animat-eurrice-s. Les différentes composantes qui confèrent à une activité une dimension ludique sont souvent mises à mal par les diverses transformations qui semblent s'imposer au monde de l'animation de façon similaire qu'au monde (pré)scolaire et, en particulier, ses deux critères principaux. Si des objectifs pédagogiques limitent l'adoption d'un second-degré, c'est aussi particulièrement vrai du critère de la décision (de participer, à tel moment, dans tel lieu, avec telles personnes, de s'arrêter ou de continuer, etc.) qui n'incombe pas - ou plus - aux enfants joueurs et reste détenue par des animat-eur-rice-s (Besse-Patin, 2018).

\section{Une dichotomie éducative structurante}

Les distinctions et les oppositions soulignées précédemment - dont la plus classique entre « jeu libre » et « jeu dirigé » - sont les signes du paradoxe auquel se confronte les animateur-rice's tout comme les enseignante's à l'école maternelle (Brougère, 2005). Sans revenir ici sur les relations complexes entre jeu et apprentissages, on tient plutôt à souligner la récurrence d'une vision binaire du jeu au prisme de l'éducation, vision qui traverse les pratiques et les représentations analysées dans les articles du dossier. Bien que les conceptualisations du jeu aient largement évolué au cours du siècle dernier, elles restent largement opposées à partir de "caractéristiques bipolaires " (Sutton-Smith, 1986, p. 3) et, notamment sous un angle moraliste, distinguant un «bon jeu » [good play] d'un « mauvais jeu » [bad play]. Comme d'autres, celle-ci remonte aux considérations philosophiques de Platon et Aristote et conduit à penser que

la séparation entre bon jeu et mauvais jeu est probablement entre le jeu qui socialise, encourage l'apprentissage, ou sa dimension cognitive, et les autres types de jeu qui sont des dangers, une nuisance dans l'espace domestique ou scolaire et pour lesquels les enfants sont envoyés dehors. (Sutton-Smith, 1986, p. 7, notre traduction) 
(1988), cette valorisation éducative du jeu - liee à une rhétorique du progrès adossée à une conception romantique de l'enfance et du jeu peut aussi s'appuyer sur une division entre un "jeu sacré » [sacred play] et un «jeu festif » [festive play] en suivant les pensées pédagogiques de Locke, Pestalozzi et Fröbel ainsi que leurs successeurs.

Quand Locke découragea ses lecteurs d'autoriser leurs enfants de jouer dans la rue et défendit l'idée qu'ils seraient mieux à l'intérieur de la maison avec des blocs alphabétiques [...], il initia ce qui allait devenir une nouvelle distinction. Maintenant, il devait y avoir un jeu éducatif. Au cours des siècles suivants, il allait devenir un nouveau type sacralisé de jeu [...]. Ce qu'il nous reste, aujourd'hui, est la notion (ou distinction) de deux types de jeu, un éducatif qui est d'une certaine façon sacré... et un autre plus festif que nous espérons confiner à l'aire de jeux, mais qui a souvent tendance à se faufiler dans l'école et perturber nos leçons. (Sutton-Smith, 1988 , p. 45, notre traduction)

Pour n'en donner que quelques exemples, Sutton-Smith, Gerstmyer et Meckley (1988) montrent, dans une étude historique la proscription de certaines pratiques ludiques enfantines, dont les jeux de bagarre [playfighting ou rough and tumble play]. Les jeux mettant en scène la guerre [war play] ou des jouets représentant des armes plus ou moins factices ont aussi suscité de nombreuses controverses dans le monde de l'éducation préscolaire (Holland, 2003 ; Beresin, 1989). Il en va de même dans le monde de l'animation où certains jeux sont promus et valorisés, quand d'autres sont mis au ban. Malgré la diffusion des écrans et du numérique, c'est le cas encore aujourd'hui des jeux vidéo qui voient leur accès limité - confisqués voire simplement interdits - parce qu'ils ne se conforment pas à la représentation idéalisée du jeu (Brougère, 1994). Les jeux pratiqués ou animés se doivent d'être "sacralisés ", en quelque sorte adoubés par des considérations éducatives qui conduisent irrémédiablement à animer ou diriger les jeux, organiser les activités, mettre en place des projets d'animation, établir un « projet pédagogique ", etc. Autant d'actes pour encadrer deux formes ludiques distinctes, une éducative (et sacrée) produite par les animat·eur·rice's, une frivole accordée aux enfants pendant des «temps libres" selon l'expression consacrée (Besse-Patin, 2014). Cette dichotomie se perpétue ainsi à travers les considérations du monde de l'animation. De fait, elle structure aussi les contributions suivantes puisque ce rapport - plus ou moins proche ou distant - à une forme éducative du jeu est une des lectures transversales possibles.

Pourtant, des dissidences ou alternatives existent, ici et là, pour conjuguer jeu et éducation selon d'autres conceptions qui renvoient à une dimension "informelle » (Roucous, 2007). Des analyses, souvent issues de pratiques expérimentales ou marginales, mettent en avant des modèles pédagogiques différents qui repositionnent la dimension de décision précisément limitée dans la très grande majorité des accueils de loisirs. On peut citer sur ce point, la « pédagogie de la décision » (Houssaye, 1995) ou la «pédagogie de la liberté» (Bataille, 2007) qui convergent pour penser des organisations à contre-pied de la forme scolaire en s'appuyant sur cette dimension non contraignante qui caractérise le loisir. Le jeu y trouve ainsi une place prépondérante (Houssaye, 2007) en tant qu'il constitue une pratique particulièrement appréciée des enfants, mais sans exclusivité puisque le bricolage, les promenades, la lecture - pour ne citer qu'eux - ou encore la simple discussion entre pairs figurent aussi comme des centres d'intérêt fort pour eux. Les cadres qui organisent les temps, les espaces et les activités sont donc volontairement réduits au minimum, ou plus précisément, ils 
relèvent d'une co-construction entre les enfants et les professionnel-le's de l'accueil. La posture d'encadrement se transforme en une posture d'accompagnement qui vise à s'assurer que chacun trouve son compte dans ce collectif qui se constitue au gré des goûts des uns et des autres, non parfois sans difficulté.

Ces approches renouent ainsi avec ce qui apparaît par contraste comme « l'informel du jeu ", même si formule peut sembler redondante. Elles se situent, et le revendiquant d'ailleurs, dans une perspective de dés-encadrement du jeu ou, plus exactement dans une réaffirmation du cadre du jeu au détriment du cadre de l'animation, pour échapper à l'antinomie structurelle entre ces deux approches. Pour autant, comme le suggère l'appellation pédagogie, la question éducative n'est pas évacuée mais revisitée. La coconstruction du cadre autrement dit des fonctionnements et modalités qui permettent de vivre ensemble ce temps de loisir, est directement le lieu d'une socialisation (Houssaye, 2005). "Les enfants y expérimentent un nouveau rapport à autrui, un rapport négocié entre individualité et regroupement, individualité et institution » (Houssaye 1995, p.14). L'éducation passe ici par l'organisation collective qui permet d'apprendre de la vie quotidienne pour reprendre le titre de l'ouvrage de Brougère et Ulmann (2009). "Sans que le jeu perde sa dimension de loisir, ou peut-être parce qu'il est loisir, il est le lieu d'apprentissage des logiques sociales adultes et plus largement du fonctionnement des relations sociales " (Brougère, 2009, p. 124). Ces accueils atypiques montrent ainsi une ouverture possible vers la reconnaissance de l'informel des situations d'apprentissage. Il s'agit là indéniablement d'un changement fondamental de perspective par rapport aux formes éducatives qu'elles soient scolaire ou animative. Elle montre qu'une compatibilité existe entre jeu et animation qui permet de réinscrire les accueils dans une perspective de loisir à condition de revoir cette pierre angulaire qu'est l'encadrement et ce qu'elle implique dans la conception de l'enfant (Roucous, 2007).

\section{Des articles dans la continuité}

De façon transversale, les articles réunis dans ce dossier reposent principalement sur des travaux portant sur l'animation avant de s'intéresser au jeu. Parce que ce dernier est un outil et un support employé par des animat-eur-rice-s, il est invoqué mais aucun texte porte avant tout sur le jeu, ni sur les usages de jeux et jouets, les éventuelles spécificités d'une culture ludique (matérielle) propre à l'animation. Par ailleurs, une variété de disciplines sont convoquées (psychologie, sciences de l'éducation, didactique, sociologie) tout comme une diversité d'approches méthodologiques employées pour explorer divers objets. Tantôt l'analyse porte sur les discours voire les représentations d'enfants, de jeunes ou d'animateurrice.s, tantôt la focale est mise sur des pratiques observées et leurs effets supposés. Ainsi, chacun des articles contribue à éclairer le croisement des deux objets par des perspectives différentes. Cela dit, il convient d'ajouter que l'ensemble des articles étudient l'animation adressée à des enfants et des jeunes et qui prend place dans des accueils de loisirs, notamment périscolaires, à une exception près. De par les relations complexes, discutées ci-dessus, entre l'animation, le loisir et l'éducation, ce n'est pas un hasard si l'on retrouve, en filigrane ou de façon explicite, l'évocation ou une discussion plus serrée des rapports de l'animation ou du jeu à l'éducation. 
49 Avant de présenter les articles réunis, nous voudrions souligner que le dossier est aussi conçu comme un ensemble dans lequel l'ordre des textes répond à la logique suivante. En rentrant par les discours et les représentations, le premier texte donne l'occasion d'appréhender les perceptions ainsi que les liens établis entre le loisir et le jeu, que ce soit par des enfants et adolescent·e's ou des animat-rice'eur's. Les deux contributions suivantes donnent à voir comment ces représentations peuvent se traduire en pratique dans des accueils périscolaires. Enfin, les deux derniers textes proposent des contrepoints en quittant la situation française pour découvrir le monde du playwork et en s'intéressant à une animation davantage inscrite dans le divertissement.

50 Ancrée dans une approche de psychologie sociale, la contribution de Stéphanie Constans, Véronique Rouyer et Emmanuèle Gardair se situe dans le domaine d'intervention privilégiée de l'animation, celui du temps libéré et des loisirs des enfants et des jeunes. En synthétisant des travaux précédents, elles proposent de comprendre les représentations et la structuration des discours que les animateurs et les animatrices ont de leur action, des fonctions de leur métier, du «public » et, plus spécifiquement, $\mathrm{du}$ jeu et du loisir. En miroir, elles s'intéressent aussi aux représentations du loisir de ces publics en distinguant enfants et adolescent.e's pour comprendre comment le jeu s'y insère de leur point de vue. L'analyse trouve sa pertinence à la fois dans l'approche plus quantitative et systématique qui permet de mettre à jour divers profils et par l'introduction du double point de vue des acteurs qui rend possible les croisements. C'est cependant la double entrée "âge" (des publics enfant et adolescent) et "genre" (pour les enfants/adolescent·e's et les professionnel·le·s) qui donne son intérêt essentiel au texte. Elle permet de faire un tableau précis et en même temps de montrer la variabilité de ces conceptions du loisir et du jeu. Animateurs et animatrices ne pensent pas le loisir et le jeu de la même façon entre eux et font varier leur regard en fonction des âges du public. Sur ce point, on peut souligner un résultat peu attendu qui souligne un plus fort intérêt des animateurs pour l'éducatif par rapport aux animatrices qui mettent davantage en avant les dimensions relationnelles et sociales du loisir. Concernant les publics, si les représentations entre enfants et adolescents se distinguent assez nettement, elles semblent peu traversées par des différences de genre, même si les auteurs invitent à la prudence sur ce dernier résultat. Pour finir, les analyses concernant plus précisément le jeu confirment sa marginalisation dans les représentations mais permet d'en éclairer les origines à la fois du côté des animateur·rice's et du côté des enfants ou adolescent qui occultent les pratiques réelles.

51 Les contributions de Vanessa Desvages-Vasselin et Simon Kechichian portent toutes les deux sur des pratiques d'animation au sein d'accueils extra-scolaires. Ces accueils se situent dans le contexte de la réforme dite des « rythmes scolaires » de 2013 en France, dans laquelle de nouveaux dispositifs accompagnés de nouvelles «activités périscolaires » ont été mis en place et animés par des équipes d'animation dans les interstices du temps scolaire. Dans les deux cas, les analyses conduisent les auteur·e·s à situer ces pratiques par rapport au système scolaire mais dans des approches et des perspectives différentes.

52 L'article de Vanessa Desvages-Vasselin se focalise en effet sur un aspect du dispositif qui visait à renforcer les liens entre le hors scolaire et le scolaire pour créer davantage de continuité éducative. L'analyse est centrée sur le cas d'un animateur qui organise un jeu sportif avec un groupe d'enfants dans le cadre de ces ateliers. Suivant les 
propositions de la didactique clinique, la «carrière " comme la "culture ludique " personnelle de l'animateur est confrontée, grâce à deux entretiens, à sa pratique réelle observée pour en comprendre les incidences sur les apprentissages recherchés. On retrouve dans ces analyses l'apparent paradoxe déjà décrit par Thin (1994) entre la culture antiscolaire, qu'avance le jeune animateur ayant rencontré des difficultés au cours de scolarité tout en revendiquant une reconnaissance éducative, et la " continuité éducative » annoncée et recherchée.

Le texte de Simon Kechichian interroge plus spécifiquement la dimension corporelle des pratiques animatives, et notamment celles qui s'annoncent "ludiques", à partir d'une enquête ethnographique menée dans plusieurs accueils périscolaires. Le choix de l'approche foucaldienne éclaire sous un nouvel angle les relations entre les usages des jeux par les animat-eur.rice's et les intentions éducatives, plus ou moins explicites, qui y sont rattachées, pour tempérer l'excitation, capter l'attention ou tenter de transmettre des apprentissages. De façon tout à fait contradictoire avec certains messages aux enfants formulés par les animateur.rice's qui affirment se distinguer de l'école, la question de la forme scolaire et plus particulièrement du poids de la discipline apparaît ici comme un modèle profondément ancré et intégré dans les pratiques. On est tenté de dire que le comble de la forme scolaire - pour réutiliser également un élément de la culture enfantine - apparait lorsque les professionnelle's font appel à ces règles ou rituels que les enfants utilisent eux-mêmes spontanément dans leurs jeux pour leur imposer un cadre qui n'a plus rien de secondaire. On en vient ainsi à se demander si c'est la dimension éducative recherchée qui impose le cadrage, ou si ne se joue pas ici davantage une volonté de rester maitre du jeu.

Les deux dernières contributions sont plus éloignées à la fois de ces accueils typiques de l'animation « enfance-jeunesse ", mais aussi du modèle « à la française » apportant par là-même de nouveaux éclairages. Elles restent en effet traversées par ce questionnement sur la forme de l'intervention des professionnelle's dans le domaine du loisir et plus précisément du jeu, ce qui les conduit comme inexorablement à rencontrer la tension entre éducation et divertissement.

La contribution de Shelly Newstead aborde un domaine quasiment inconnu en langue française, le playwork. À partir de l'analyse documentaire de sources primaires, elle nous plonge dans les méandres d'un métier qui rencontre, encore malgré ces 70 années d'existence, de nombreuses difficultés pour se définir et, incidemment, se faire reconnaître notamment à côté d'autres professions qui interviennent auprès d'enfants. Issu des terrains d'aventure, expériences déjà méconnues en France (Raveneau, à paraître), le playwork s'est développé au Royaume-Uni à partir des années 1960 en s'écartant du playleadership apparu aux États-Unis au début du $20^{\mathrm{e}}$ siècle (Cavallo, 1981). Si cette contribution offre de nombreux parallèles avec l'animation française, dont la recherche récurrente d'une reconnaissance professionnelle ou la présence d'une «idéologie du don» (Besnard, 1980, p. 107-108), elle donne surtout à voir un autre rapport au jeu et aux enfants que le playwork a tenté de définir à travers plusieurs approches - au fil des périodes - discutées et critiquées. L'approche internationale permet ainsi d'éclairer sous un nouveau jour - critique - l'animation à la française et le peu de cas fait du jeu des enfants. Dans ce panorama, un rapprochement apparaît avec les ludothèques, ces structures qui se situent délibérément quelque peu à la marge du monde de l'animation mais qui fondent toutes leurs pratiques sur le jeu avec une posture d'accompagnement très proche de celle du playwork (Roucous \& Haberbusch, 
2010 ; Brougère et al., 2016). Ces convergences n'ont cependant pas permis de résoudre les problèmes de traduction du registre du playwork. De fait, dans les dénominations de l'équipement et du métier - ludothèque et ludothécaire - « le travail du jeu » n'est pas mis en valeur au profit de celui de gestionnaire du lieu et des objets. Lorsque d'autres termes sont avancés l'animation réapparaît avec le titre d' " animateur jeu », mais avec les mêmes réserves et défiances que celles des playworkers vis-à-vis des autres métiers de l'enfance.

Pour finir, le texte de Sophie Corbillé conduit le lecteur dans un autre monde du jeu, à certains égards, un peu surréaliste. Avec une approche ethnographique très ciblée, elle explore un parc de loisir à thème qui a pour particularité de reproduire le monde adulte pour le donner à jouer aux enfants. Le souci d'hyperréalisme conduit les organisateurs à faire appel à des enseignes commerciales réelles pour installer des espaces qui reproduisent leur activité à échelle réduite. Le parc reproduit surtout toute la logique économique qui lie travail, rémunération, consommation, système monétaire et bancaire et loisir, tout en intégrant des services publics qui organisent la société moderne. Les enfants sont ainsi invités à vivre dans cette mini-ville mais aussi à y travailler pour pouvoir profiter de l'offre de consommation ou de loisir. La dimension artificielle situe d'emblée cette proposition dans un contexte de loisir, et plus spécifiquement encore de divertissement. Mais les proximités avec les structures plus classiques du monde de l'animation sont étonnement fortes. D'une part, la question éducative reste prégnante et très clairement affichée voire revendiquée par les organisateurs pour qui "jouer à travailler ", c'est " apprendre à travailler ", avec tout ce que recouvre le système du travail dans le modèle économique proposé. D'autre part, les observations révèlent les formes d'organisation et de cadrage très stricts qui sont en partie liées à ces intentions éducatives. Le texte trouve surtout sa pertinence dans les analyses subtiles de ce que signifie, pour un adulte ici professionnelle, de donner à jouer à un enfant dans une forme de jeu symbolique assez inhabituelle. Comment tenir son multiple rôle de stimulateur, d'organisateur, de garant ou encore d'arbitre, voire de juge? Le texte donne par ailleurs à réfléchir sur la difficulté, voire l'impossibilité, de construire et de suivre un script de jeu tel que peuvent le proposer d'autres formes pédagogiques. L'animation, et particulièrement celle d'un jeu, se doit de maintenir cette incertitude qui rentre en concurrence avec le script précis et l'encadrement posé et cela demande un travail émotionnel conséquent aux intervenante-s. À plusieurs égards, et malgré une distance aux contextes habituels de l'animation, les réflexions développées permettent de relire à nouveaux frais le travail ordinaire des animateur.rice's.

\section{BIBLIOGRAPHIE}

ANZIEU D. \& MARTIN J.-Y. (1997), La dynamique des groupes restreints, $11^{\mathrm{e}}$ éd., Paris, Presses

Universitaires de France. 
AUGUSTIN J.-P. \& GILLET J.-C. (1997), « Pour un mouvement de recherche et d'expérimentation sur l'animation », Agora débats/jeunesses, 8, pp. 129-132.

AUGUSTIN J.P. \& GILLET J.-C. (2000), L'animation professionnelle. Histoire, acteurs, enjeux, Paris, L'Harmattan.

AUGUSTIN J.-P. \& ION J. (2017), Loisirs des jeunes : 120 ans d'activités éducatives et sportives, réédition, Paris, La Documentation française.

BATAILLE J.-M. (2007), Enfants à la colo. Courcelles, une pédagogie de la liberté, Marly-le-Roi, INJEP. BECKER H. S. (1988), Les mondes de l'art, Paris, Flammarion.

BERESIN A.R. (1989), « Toy War Games and the Illusion of Two-Sided Rhetoric », Play \& Culture, 2, pp. 218-224.

BERGER É., ABADIE F. \& WITTMAN S. (dir.). (2018), L'éducation non formelle : chance ou défi pour le travail de jeunesse ?, Paris/Berlin, OFAJ. https://www.ofaj.org/resources/flipbooks/texte-de-travail_30/ files/assets/common/downloads/publication.pdf ?uni =c970a76d1f69fee08c2b444954c7034b BESNARD P. (1972), «Éléments pour une théorie du système d'animation », Cahiers de l'animation, 1, pp. 29-35.

BESNARD P. (1978), « L'animation socioculturelle (Recherches) », Revue française de pédagogie, 44, pp. 129-142.

BESNARD P. (1980), Animateur socioculturel. Une profession différente ?, Issy-les-Moulineaux, ESF. BESNARD P. (1985), L'animation socioculturelle, Paris, Presses Universitaires de France.

BESSE L., CHATEIGNER F. \& IHADDADÈNE F. (2017), « L'éducation populaire », Savoirs, 42, pp. 11-49.

BESSE-PATIN B. (2014), « Du jeu dans la professionnalisation de l'animation », Animation, territoires et pratiques socioculturelles, 6, pp. 93-104.

BESSE-PATIN B. (2018), « Loisir et éducation. La formalisation éducative des jeux dans un accueil périscolaire ", Revue française de pédagogie, 204, pp. 5-16.

BESSE-PATIN B. (2019), Jeu et animation. Ethnographie des formalisations éducatives du loisir des enfants, Thèse de doctorat, Villetaneuse, Université Paris 13.

BORDES V. (2007), « La place des animateurs au sein de l'intervention sociale : quelle formation pour quelles missions ?», Pensée plurielle, 15, pp. 101-109.

BORDES V. (2008), « L'impossible professionnalisation de l'animation », Les cahiers de l'implication, 2, pp. 81-90.

BRET D. (2008), « L'enseignant d'EPS. Animateur, technicien, ingénieur ou concepteur », Recherche \& formation, 57, pp. 141-152.

BROUGÈRE G. (1992), Le jouet. Valeurs et paradoxes d'un petit objet secret, Paris, Autrement. BROUGÈRE G. (1994), « Le jeu entre domestication et idéalisation », in M. Glaumard-Carré (dir.), Plaisirs d'enfances. L'enfant, acteur de lien social, Paris, Syros, pp. 31-41.

BROUGÈRE G. (1995), Jeu et éducation, Paris, L'Harmattan. BROUGÈRE G. (2005), Jouer/Apprendre, Paris, Economica. BROUGÈRE G. (2010), « Formes ludiques et formes éducatives », in J. Bédard J. \& G. Brougère (dir.), Jeu et apprentissage : quelles relations ?, Sherbrooke, Éditions du CRP, pp. 43-62. 
BROUGÈRE G. (2009), « Loisirs et apprentissage », in G. Brougère. \& A.-L. Ulmann (dir.), Apprendre de la vie quotidienne, Paris, Presses Universitaires de France, pp. 119-129.

BROUGÈRE G. \& ULMANN A.-L. (dir.). (2009), Apprendre de la vie quotidienne, Paris, Presses Universitaires de France.

BROUGÈRE G., ROUCOUS N., BESSE-PATIN B. \& CLAUDE V. (2016), Une boîte pour jouer : pratiques et discours autour d'objets recyclés, Rapport d'évaluation, Paris, Université Paris 13 - Sorbonne Paris Cité, https://halshs.archives-ouvertes.fr/halshs-01361254/document BURMAN E. (2008), Deconstructing Developmental Psychology, $2^{\mathrm{e}}$ éd., London, Routledge. CAMUS J. \& LEBON F. (2015), « Introduction. Pour une sociologie de l'animation », in J. Camus \& F. Lebon (dir.), Regards sociologiques sur l'animation, Paris, La Documentation française, pp. 9-13. CARLETTI M. (2014), «L'expression “animation" dans les discours : sens et catégorisation », Animation, territoires et pratiques socioculturelles, 7, pp. 1-14.

CAVALLO D. (1981), Muscles and Morals : Organized Playgrounds and Urban Reform, 1880-1920, Philadelphia, University of Pennsylvania Press.

CHIVot D. (2004), « Télévision », Études, 400, 5, pp. 681-683.

CLAPARÈDE É. (1920), Psychologie de l'enfant et pédagogie expérimentale, 8 e éd., Genève, Kundig.

CLARK C. \& PINCH T. (1995), The Hard Sell : The Language and Lessons of Street-wise Marketing, Londres, HarperCollins.

COURTOIS G. (1936), Jeux à thème chrétien, Paris, O-gé-O.

Della CROCE C., LIBOIS J. \& MAWAD R. (2011), Animation socioculturelle. Pratiques multiples pour un métier complexe, Paris, L'Harmattan.

DANSAC C. \& VACHÉE C. (2016), « Les fonctions professionnelles de l'animateur : Un modèle à cinq dimensions comme repère pour l'analyse des compétences et de l'action ", in M. H. Khadraoui (dir.), Les métiers de l'animation et de la médiation et les transformations sociales, Tunis. https://hal-univ-tlse2.archives-ouvertes.fr/hal-01466424v2/document

DANSAC C. \& VACHÉE C. (2018), « Une fonction récréative dans l'animation socioculturelle? Réflexions à partir d'un modèle théorique et de données empiriques portant sur l'animation jeunesse ", Colloque international de l'ISIAT, présenté à Jeu et enjeux pour l'animation socioculturelle, Bordeaux. https://hal.archives-ouvertes.fr/hal-01883268/document DESSERTINE D. \& MARADAN B. (1998), « La socialisation des enfants hors de l'école : la belle époque des patronages (1900-1939) », Éducation et sociétés, 2, pp. 119-134.

DESSERTINE D. \& MARADAN B. (2001), L'âge d'or des patronages, 1919-1939 : la socialisation de l'enfance par les loisirs, Paris, La Documentation française.

DUMAZEDIER J. (1962), Vers une civilisation du loisir ?, Paris, Seuil.

ELIAS N. \& DUNNING É. (1994), Sport et civilisation. La violence maîtrisée, Paris, Fayard.

GILLET J.-C. (1995), Animation et animateurs. Le sens de l'action, Paris, L'Harmattan.

GILLET J.-C. (dir.). (2005a), L'animation dans tous ses états (ou presque), Paris, L'Harmattan.

GILLET J.-C. (2005b), «L'animation et ses analogies : perspectives internationales », Agora débats/

jeunesses, 39, pp. 12-24.

GILLET J.-C. (2006), L'animation en questions, Ramonville Saint-Agne, Érès. 
GILLET J.-C. (2008), Des animateurs parlent : militance, technique, médiaction, Paris, L'Harmattan.

GILLET J.-C. (2010), « Petit précis historique sur l'ISIAT », Cahier ADES, 7, pp. 21-26.

GOFFMAN E. (1991), Les cadres de l'expérience, Paris, Minuit.

GRAM M. (2003), Grounds to Play: Culture-Specific Ideals in the Upbringing of Children in France, Germany, and the Netherlands, Bern, Peter Lang.

GRANDCLÉMENT C. (2004), « Climatiser le marché. Les contributions des marketings de l'ambiance et de l'atmosphère ", ethnographiques.org, 6. http://www.ethnographiques.org/2004/ Grandclement

GREFFIER L. (2013), « L'animation en France, tentative de définition d'un système complexe », Autour de l'animation sociale et socioculturelle en France et en Espagne, Bordeaux, Carrières sociales, pp. 15-31. https://books.openedition.org/cse/389 ?lang =fr.

GUILLEN É. (1942), Le Livre des jeux. 600 jeux d'éclaireurs, $7^{e}$ éd., Paris, L'Arc tendu.

HENNION A. \& MÉADEL C. (1986), « La rhétorique de la radio, ou comment garder l'auditeur à l'écoute ", Vibrations. Musiques, médias, société, 3, pp. 60-75.

HOLLAND P. (2003), We Don't Play with Guns Here : War, Weapon and Superhero Play in the Early Years, Maidenhead, Open University Press.

HOUSSAYE J. (1989), Le Livre des colos. Histoire et évolution des centres de vacances pour enfants, Paris, La Documentation française.

HOUSSAYE J. (1995), Et pourquoi que les colos, elles sont pas comme ça? Histoires d'ailleurs et d'Asnelles, Vigneux, Matrice.

HOUSSAYE J. (1998), « Le centre de vacances et de loisirs prisonnier de la forme scolaire », Revue française de pédagogie, 125, pp. 95-107.

HOUSSAYE J. (2005), C'est beau comme une colo. La socialisation en centre de vacances, Vigneux, Matrice.

HOUSSAYE J. (2007), « Jeux libres et socialisation », in HOUSSAYE J. (dir.), Colos et centres de loisirs : recherches, Vigneux, Matrice, pp. 173-196.

LABOURIE R. (1978), Les institutions socio-culturelles. Les mots-clés, Paris, Presses Universitaires de France.

LABOURIE R. (2008), « De l'éducation populaire à l'animation », in F. Lebon , P. Moulinier, J.-C. Richez \& F. Tétard (dir.), Un engagement à l'épreuve de la théorie. Itinéraires et travaux de Geneviève Poujol, Paris, L’Harmattan, pp. 47-49.

LAFORTUNE J.-M., AUGUSTIN J.-P., BÉLANGER P. \& GILLET J.-C. (2010), « Vers un système d'animation socioculturelle : défis actuels et synergies internationales », Animation, territoires et pratiques socioculturelles, 1, pp. 1-12.

LEBON F. (2005), Une politique de l'enfance. Du patronage au centre de loisirs, Paris, L'Harmattan.

LEBON F. (2008), « Des intellectuels sécants ? Enquête sur les auteurs des Cahiers de l'animation », in F. Lebon, P. Moulinier, J.-C. Richez \& F. Tétard (dir.), Un engagement à l'épreuve de la théorie. Itinéraires et travaux de Geneviève Poujol, Paris, L’Harmattan, pp. 71-91.

LEBON F. (2009), Les animateurs socioculturels, Paris, La Découverte. 
LEBON F. \& LIMA L. (2011), « Les difficultés au travail dans l'animation », Agora débats/jeunesses, 57, pp. 23-36.

LEBON F. \& DE LESCURE E. (dir.), (2016), L'éducation populaire au tournant du XXI ${ }^{e}$ siècle, Vulaines-surSeine, Croquant.

LEE DOWNS L. (2009), Histoire des colonies de vacances de 1880 à nos jours, Paris, Perrin.

LEE DOWNS L. (2011), « De la colonie « politique » à la colonie « éducative » : le cas de la colonie communiste d'Ivry-sur-Seine, 1925-1960», in L. Greffier (dir.), Les vacances et l'animation : espaces de pratiques et de représentations, Paris, L'Harmattan, pp. 131-144.

LEROUX P. \& RIUTORT P. (2006), « La consécration de l'animateur. Appréciation d'un métier et affirmation d'une position : les métamorphoses de Thierry Ardisson », Réseaux, 139, pp. 219-248. LEWIN K. (1959), Psychologie dynamique. Les relations humaines, Paris, Presses Universitaires de France.

LEWIN K. \& LIPPITT R. O. (1938), « An Experimental Approach to the Study of Autocracy and Democracy : A Preliminary Note ", Sociometry, 1, 3/4, pp. 292-300.

LICOPPE C. (2006), « La construction conversationnelle de l'activité commerciale. « Rebondir » au téléphone pour placer des services », Réseaux, 135-136, pp. 125-159.

Limbos É. (1971), L'Animateur socio-culturel. Formation et auto-formation, méthodes et techniques, Paris, Fleurus.

LIMBOS É. (1974), Pratique et instruments de l'animation socio-culturelle, Paris, Fleurus.

LIPPITT R. O. \& WHITE R. K. (1943), «The « Social Climate » of Children's Groups », in R.G. Barker, J. S. Kounin \& H. F. Wright, (eds.), Child Behavior and Development : A Course of Representative Studies, New York, McGraw-Hill, pp. 485-508.

MAUGER G. (2015), «L'animation socioculturelle : limites externes et clivages internes », in J. Camus \& F. Lebon (dir.), Regards sociologiques sur l'animation, Paris, La Documentation française, pp. 159-167.

MÉADEL C. (1984), «Ethnographie de l'antenne, Le travail des gens de radio », Réseaux, 9, pp. 77-98.

MEHL D. (1994), « La télévision, le public et l'animateur », Communication \& Langages, 99, pp. 20-34.

MOSER H., MULLER E., WETTSTEIN H. \& WILLENER A. (2004), L'animation socioculturelle. Fondements, modèles et pratiques, Genève, IES. https://books.openedition.org/ies/1482

MOULINIER P. (1978), « Les animateurs vacataires de l'indépendance pédagogique », Les Cahiers de l'animation, 22, pp. 29-35.

MOULINIER P. (2008), «Sur les fondements d'une politique. Retour sur le débat culturel/ socioculturel », in F. Lebon , P. Moulinier, J.-C. Richez \& F. Tétard (dir.), Un engagement à l'épreuve de la théorie. Itinéraires et travaux de Geneviève Poujol, Paris, L'Harmattan, pp. 175-188.

NDIAYE A. (2007), « Sociographie d'un rapport au temps comme fondement de la culture professionnelle. L'exemple des animateurs socioculturels », SociologieS. https://

journals.openedition.org/sociologies/1232

OBERTI A. (2008), Professionnels de jeunesse et d'éducation non formelle, Paris, INJEP.

OTTAVI D. (2001), De Darwin à Piaget. Pour une histoire de la psychologie de l'enfant, Paris, CNRS. 
POMBET T. (2014), « Un espace de loisirs à l'hôpital. Entre promotion de la bienveillance et régulation de la générosité ", Les Sciences de l'éducation - Pour l'Ère nouvelle, 47, 3, pp. 41-61. PoujoL G. (1972), « Recherche sur l'animation en France », Cahiers de l'animation, 1, pp. 29-35. POUjoL G. (1989), Profession : animateur, Toulouse, Privat.

PRÉCAS N. (1984), « Pour une approche anthropologique du métier de l'animateur », Cahiers de l'animation, 44-45, pp. 113-119.

PRÉCAS N. (2004), « Regards sur le métier d'animateur », Agora débats/jeunesses, 36, pp. 30-38.

RAVENEAU G. (à paraître), « Les terrains d'aventure en France dans les années 1970 ou la contestation en acte de la notion d'équipement socioculturel ", in L. Besse (dir.), Des lieux pour l'éducation populaire (1930-1970). Histoire des équipements socioculturels, Paris.

REMOUSSENARD-POURQUIER C. et DITTER J.-G. (2015), « De la coordination au leadership : le rôle de l'animateur de cluster ", Revue d'économie industrielle, 152, pp. 39-65.

RENAUT A. (2002), La libération des enfants. Contribution philosophique à une histoire de l'enfance, Paris, Bayard.

ROGERS C. R. (1966), Le développement de la personne, Paris, Dunod.

Roucous N. (2007), « Les loisirs de l'enfant ou le défi de l'éducation informelle », Revue française de pédagogie, 160, pp. 63-73.

ROUCOUS N. (2010), « Du jeu et des vacances en colonie, une évidence en question », in J. Houssaye (dir.), Colos et centres de loisirs : institutions et pratiques pédagogiques, Vigneux, Matrice, pp. 205-219.

ROUCOUS N. \& HABERBUSCH N. (2010), «Culture enfantine, jouets et jeu symbolique en ludothèque ", in S. Rayna \& G. Brougère (dir.), Jeu et cultures préscolaires, Lyon, INRP, pp. 191-224. ROUCOUS N. \& ADAM D. (2016), «L'éducation populaire aux prises avec le loisir des enfants », in F. Lebon \& E. de Lescure, (dir.), L'éducation populaire au tournant du XXI ${ }^{e}$ siècle, Vulaines-sur-Seine, Croquant, pp. 63-77.

schmitt A. \& Boulogne A. (1955), La cure de santé et les jeux des enfants, Paris, Scarabée.

SEGRESTAN P. \& GOUJU J.-L. (2013), Panorama des situations professionnelles du champ de l'animation, Paris, CAFEMAS.

SIMONOT M. (1974), Les animateurs socio-culturels. Étude d'une aspiration à une activité sociale, Paris, Presses Universitaires de France.

STOIAN E. (2011), « Au-delà et en-deçà de l'écran. Le double engagement des animateurs de chat rose au travail », ethnographiques.org, 23, https://www.ethnographiques.org/2011/Stoian SUE R. (1982), Vers une société du temps libre ?, Paris, Presses Universitaires de France.

SUE R. (1988), Le loisir, Paris, Presses Universitaires de France.

SUTTON-SMitH B. (1970), «Psychology of Childlore : The Triviality Barrier », Western Folklore, 29, 1, pp. 1-8.

SUTTON-SMith B. (1986), « The Spirit of Play », in G.G. Fein et M.S. Rivkin (eds.), The Young Child at Play: Review of Research, vol. 4, Washington, NAEYC, pp. 3-15. 
SUTTON-SMitH B. (1988), « The Struggle between Sacred Play and Festive Play », in D. Bergen (ed.), Play as Medium for Learning and Development : A Handbook of Theory and Practice, Portsmouth, Heinemann, pp. 45-47.

SUTTON-SMITH B., GERSTMYER J. \& MEKLEY A. M. (1988), « Playfighting as Folkplay amongst Preschool Children », Western Folklore, 47, 3, pp. 161-176.

THIN D. (1994), «Travail social et travail pédagogique. Une mise en cause paradoxale de l'école », in G. Vincent (dir.), L'éducation prisonnière de la forme scolaire. Scolarisation et socialisation dans les sociétés industrielles, Lyon, Presses Universitaires de Lyon, pp. 51-71.

VINCENT G. (1980), L'école primaire française. Étude sociologique, Lyon, Presses Universitaires de Lyon. DE VISSCHER H. (2014), « L'animateur dans tous ses états », Cahiers Internationaux de Psychologie Sociale, 104, pp. 763-770.

DE VISSCHER P. (2011), « Articulations et emboîtements des pratiques d'animation de groupes restreints - Un vade-mecum », Cahiers Internationaux de Psychologie Sociale, 89-90, pp. 163-176.

WITTGENSTEIN L. (2004 [1953]) Recherches Philosophiques, Paris, Gallimard.

YONNET P. (1999), Travail, loisir. Temps libre et lien social, Paris, Gallimard.

\section{NOTES}

1. Il faut souligner ici le développement exponentiel de ce dernier usage du mot en association avec des images. L'animation désigne de plus en plus ce domaine artistique qui se développe autour des films d'animation et des techniques du dessin animé à tel point que cet usage tend à supplanter celui qui fait l'objet de ce numéro.

2. Ortolang du centre national de ressources textuelles et lexicales httpshttps://www.cnrtl.fr/ definition/animation://www.cnrtl.fr/definition/animation)

3. Un baccalauréat professionnel portant la mention « animation - enfance et personnes âgées » vient d'être créé par arrêté du 22 juillet 2019.

4. Pierre Besnard, Raymond Labourie, Pierre Moulinier, Geneviève Poujol, Michel Simonot, Françoise Tétard, parmi d'autres.

5. Une partie des chercheurs est directement issue du monde professionnel et militants de l'éducation populaire et ont poursuivi un parcours universitaire; notamment sous l'égide de Joffre Dumazedier. De la même façon que l'éducation populaire, les travaux et les recherches sur l'animation ont été largement réalisés par des personnes issues de ce monde ou dites « indigènes » (voir Lebon, 2008 ; Besse et al., 2017).

6. Ce néologisme repris de Ndiaye (2007) est construit en écho aux expressions plus classiques de "pratiques éducatives » et " pratiques loisives », dont il permet précisément de se distinguer, ce qui est particulièrement utile lorsqu'on cherche comme ici à saisir la singularité du domaine

7. Par l'intermédiaire de l'IUT de l'université Bordeaux Montaigne et de sa filière Carrières sociales dont est issu l'ISIAT (Institut Supérieur d'Ingénieurs-Animateurs Territoriaux), pôle qui continue d'organiser des colloques sur l'animation tous les ans. Le RIA est aussi à l'origine de la revue internationale Animation, Territoires et animation socioculturelle.

8. D'autres auteur'e-s ont pu appréhender ou investir dans l'animation d'autres fonctions sociales jusqu'à réinterroger ce modèle pour y intégrer deux nouvelles fonctions (Dansac et Vachée, 2016) voire interroger l'absence - ou la disparition - d'une fonction récréative (Dansac et Vachée, 2018), pourtant présente chez Besnard (1980). 
9. Probablement que ces évolutions et ces changements réguliers ont un lien avec une « impossible professionnalisation » de l'animation (Bordes, 2008).

10. Après le terme de « surveillant·e ", les moniteurs sont l'appellation consacrée à cette époque avant l'apparition de la dénomination actuelle.

\section{RÉSUMÉS}

Ce texte introductif au dossier vise à cerner l'articulation entre animation et jeu pour l'éclairer et en interroger les enjeux et les limites. En partant des usages communs du terme d'animation qui laissent apparaître des convergences entre des domaines en apparence éloignés, les données scientifiques réorientent les analyses sur le seul champ de l'animation des loisirs pour en montrer à la fois le flou et les tensions. Outre les débats sur les contenus et les délimitations du domaine, l'articulation avec le loisir en tant que forme sociale se révèle être le point de cristallisation d'une double valence entre l'éducatif et le divertissement dans laquelle la question $\mathrm{du}$ jeu prend sa source. Après avoir montré l'émergence des pratiques ludiques avec la focalisation des débuts de l'animation sur les enfants, on en dévoile toutes les conséquences en lien avec l'orientation éducative de l'animation à la française. La présentation se focalise ainsi sur la mise en forme animative caractéristique des accueils de loisir en France pour montrer son inspiration forte de la forme éducative scolaire laissant par ricochet de côté la forme ludique qui permettrait d'inscrire les pratiques davantage dans le loisir. Les textes proposés dans le dossier rejoignent ces analyses des liens entre animation et jeu, mais aussi entre jeu et éducation, que ce soit à partir des représentations ou des pratiques, dans des accueils périscolaires ou plus directement de divertissement, en France ou dans d'autres pays.

This introductory text and more broadly this issue aims to identify the articulation between animation and play in order to shed light on it and question its stakes and limits. Starting from the common uses of the term animation, which reveal convergences between seemingly distant domains, scientific data redirect analyses to the sole field of leisure animation to show both the blur and the tensions. In addition to the debates on the contents and boundaries of the field, the articulation with leisure as a social form is proving to be the point of crystallization of a double valence between education and entertainment in which the question of play takes its source. After showing the emergence of playful practices with the focus of the early days of animation on children, we disclose all the consequences in relation to the educational orientation of French animation. The presentation thus focuses on the animative format characteristic of leisure activities in France to show its strong inspiration from the educational school form, leaving aside the playful form which would allow practices to be more in keeping with leisure. The proposed texts in the issue join these analyses of the links between animation and play but also between play and education, whether based on representations or practices, in extracurricular or more directly entertainment facilities, in France or in other countries.

\section{INDEX}

Keywords : entertainment, leisure, education, leisure facilities, school form, play form

Mots-clés : animation, loisir, éducation, accueils de loisir, forme scolaire, forme ludique 
AUTEURS

BAPTISTE BESSE-PATIN

Experice, Université Paris 13

NATHALIE ROUCOUS

Experice, Université Paris 13 